\title{
Neutrophil Margination, Sequestration, and Emigration in the Lungs of L-selectin-deficient Mice
}

\author{
Nicholas A. Doyle, ${ }^{\star \ddagger}$ Sabrina D. Bhagwan, ${ }^{\star}$ Barbara B. Meek, ${ }^{\star}$ Gregory J. Kutkoski, ${ }^{\star}$ Douglas A. Steeber, ${ }^{\S}$ Thomas F. Tedder, \\ and Claire M. Doerschuk ${ }^{\star \neq}$ \\ *Physiology Program, Department of Environmental Health, Harvard School of Public Health, Boston, Massachusetts $02115 ;{ }^{\ddagger}$ the \\ Herman B Wells Center for Pediatric Research, the Section of Pulmonology and Intensive Care, Department of Pediatrics and the \\ Department of Anatomy, Indiana University, Indianapolis, Indiana 46202; ${ }^{\S}$ Department of Immunology, Duke University, Durham, \\ North Carolina 27710
}

\begin{abstract}
These studies tested the hypothesis that L-selectin plays a role in neutrophil traffic in the lungs, particularly in neutrophil margination, sequestration, and emigration, using L-selectin-deficient mice. No defect in neutrophil margination within either capillaries or arterioles and venules was observed in uninflamed lungs of L-selectin-deficient mice. The initial rapid sequestration of neutrophils within the pulmonary capillaries $1 \mathrm{~min}$ after intravascular injection of complement fragments was not prevented. In contrast, L-selectin did contribute to the prolonged neutrophil sequestration ( $\geq 5 \mathrm{~min}$ ). Interestingly, neutrophil accumulation within noncapillary microvessels required L-selectin at both 1 and 5 min after complement injection. During bacterial pneumonias, L-selectin played a role in neutrophil accumulation within noncapillary microvessels in response to either Escherichia coli or Streptococcus pneumoniae and within capillaries in response to E. coli but not S. pneumoniae. However, L-selectin was not required for emigration of neutrophils or edema in response to either organism. These studies demonstrate a role for L-selectin in the prolonged sequestration of neutrophils in response to intravascular complement fragments, in the intracapillary accumulation of neutrophils during $E$. coli-induced pneumonia, and in the accumulation of neutrophils within noncapillary microvessels when induced by either intravascular complement fragments or bacteria within the airways. (J. Clin. Invest. 1997. 99:526533.) Key words: complement fragments $•$ bacterial pneumonia $•$ adhesion molecules $\cdot$ leukocytes $\cdot$ pulmonary inflammation $•$ knockout mice
\end{abstract}

\section{Introduction}

L-selectin, a member of the selectin family of adhesion molecules that is expressed on leukocytes, mediates rolling of leukocytes along the postcapillary venular endothelium of the

Address correspondence to Claire M. Doerschuk, M.D., Harvard School of Public Health, I-305, 665 Huntington Avenue, Boston, MA 02115. Phone: 617-432-1706; FAX: 617-432-3468; E-mail: cdoersch@ hsph.harvard.edu

Received for publication 24 June 1996 and accepted in revised form 21 November 1996.

J. Clin. Invest.

(C) The American Society for Clinical Investigation, Inc. 0021-9738/97/02/0526/08 \$2.00

Volume 99, Number 3, February 1997, 526-533 systemic circulation (1-3). It plays an important role in neutrophil-mediated injury or leukocyte emigration in ischemia/ reperfusion injury of the cat myocardium (4), thioglycolateinduced neutrophil emigration into the peritoneum and skin (57 ), and the accumulation of leukocytes around islets in insulindependent diabetes in mice (8). L-selectin may also mediate the infiltration of leukocytes in renal allograft rejection (9). In the lungs, L-selectin appears critical in mediating rolling of leukocytes within pulmonary venules induced by the placement of a pleural window (10). L-selectin is also required for full expression of lung injuries induced by $\mathrm{IgG}$ immune complexes or cobra venom factor $(11,12)$. The functions of L-selectin in these inflammatory diseases have been studied using anti-L-selectin antibodies, L-selectin-IgG chimeras, and fucoidin in combination with studies using anti-P-selectin antibodies.

The pulmonary capillaries contain a large pool of marginated neutrophils, as measured by an increase in the concentration of neutrophils to red blood cells $(\mathrm{RBCs})^{1}$ within pulmonary capillary blood of 60 to 100 times that found in the systemic blood $(13,14)$. In contrast to margination in the systemic circulation where selectin-mediated rolling is the mechanism, the primary mechanism through which the pulmonary intracapillary pool forms is thought to be due to the complex geometry of the capillary bed. This bed consists of multiple interconnecting segments having diameters that are usually smaller than that of the spherical neutrophils (15). Because neutrophils require longer than RBCs to deform and pass through these narrow segments, their transit time is lengthened, resulting in a increase in their concentration within the pulmonary capillary blood $(13,14,16)$. However, adhesion molecules may also play a role in lengthening the capillary transit time of neutrophils. Although Yoder and colleagues have shown that CD11/CD18 is not required for the formation of the marginated pool in normal dogs (17), the role of selectins has not been evaluated. Their potential importance is suggested by their ability to mediate loose, rapidly cycling interactions with their respective ligands. E-selectin is not a candidate, as it is not constitutively expressed (1). P-selectin is also not expressed on pulmonary capillary endothelium of normal rats, mice, and rabbits (18). However, L-selectin remains a possibility.

Complement fragments and other intravascular inflammatory mediators induce neutrophil sequestration within the pulmonary capillaries, defined as an increase in the number of intravascular neutrophils induced by inflammatory mediators above the number present due to normal margination. Seques-

1. Abbreviations used in this paper: EVA, extravascular albumin; $\mathrm{RBC}$, red blood cell; ZAP, zymosan-activated plasma. 
tration occurs through mechanisms that require at least two sequential steps. First, rapid sequestration of neutrophils, which results in neutropenia within 0.5 min of infusion of complement fragments, occurs through a process that does not require CD11/CD18 (19). The mechanism is thought to involve a stimulus-induced decrease in the neutrophil's ability to deform that may delay or prevent neutrophils from passing through the capillary bed $(14,19-21)$. Second, prolonged sequestration of these intracapillary neutrophils for more than a few minutes does require CD11/CD18-mediated interactions with the endothelium (19). The role of L-selectin in either the initial sequestration of neutrophils or in maintaining the sequestered neutrophils within the capillary bed has not been evaluated. The observation that L-selectin was required for full induction of injury by cobra venom factor, a neutrophil-mediated lung injury that requires adhesion but not migration, suggests that L-selectin may play a role (11).

Neutrophil emigration into the lung parenchyma in response to stimuli within the alveolar space occurs through at least two different adhesion pathways. For example, neutrophil emigration induced by Escherichia coli requires CD11/ CD18 while emigration elicited by Streptococcus pneumoniae does not (22). In lung injury induced by IgG immune complexes, a CD11/CD18-mediated injury, L-selectin is necessary for neutrophil emigration and full expression of injury (12). However, the role of L-selectin in CD11/CD18-dependent and -independent pathways of emigration during bacterial pneumonias has not been evaluated.

Recently, L-selectin-deficient mice have been generated $(23,24)$. These animals develop and reproduce normally and have normal circulating mononuclear and neutrophil counts up to the age of $14 \mathrm{wk}$. However, lymphocyte homing to peripheral and mucosal lymphoid tissue is reduced while homing to the spleen is increased $(23,24,25)$. These mice have no defect in the leukocyte rolling that is present immediately after exterioration of the cremaster muscle. However, the increased leukocyte rolling that occurs after $20 \mathrm{~min}$ is completely prevented, as well as TNF- $\alpha$-induced rolling (26). These mice have a complete defect in thioglycolate-induced emigration of neutrophils into the peritoneum after $1 \mathrm{~h}$ and a partial defect of $50-65 \%$ at 4,24 , and $48 \mathrm{~h}$, as well as $70-80 \%$ reductions in the numbers of lymphocytes and macrophages at 24 and $48 \mathrm{~h}$ (24), indicating that L-selectin is required for optimal recruitment of all leukocyte types. These mice also demonstrate impairments of $60-80 \%$ in tissue swelling induced by delayedtype hypersensitivity reactions induced by either oxazolone or sheep RBCs, as well as defects in lymphocyte accumulation within the draining lymph nodes (24). Finally, L-selectin deficiency protected against death induced by endotoxemia (24). These studies demonstrate an important role for L-selectin in the response of leukocytes to many different types of inflammatory injuries.

This study tested the hypothesis that L-selectin is an important mediator of neutrophil responses within the pulmonary microvasculature using L-selectin deficient and wild type mice. The role of L-selectin was examined in neutrophil margination within the pulmonary capillaries of mice without experimentally induced inflammation, in neutrophil sequestration within the capillaries and noncapillary microvasculature induced by intravascular complement fragments, and in neutrophil emigration induced by either E. coli or $S$. pneumoniae within the distal airways of the lungs.

\section{Methods}

$L$-selectin deficient mice. Mice deficient in L-selectin were generated by mutation of the L-selectin gene in embryonic stem cells using homologous recombination as previously described (23) and backcrossed with C57BL/6 mice for at least four generations. The mutation resulted in mice that were completely deficient in L-selectin expression but not in P- or E-selectin. This phenotype was confirmed using immunohistochemistry to evaluate the expression of L-selectin on neutrophils in the lung. Age-matched wild type mice used as controls were C57BL/6 mice purchased from Harlan Sprague Dawley, Inc. (Indianapolis, IN).

Neutrophil margination in the pulmonary capillaries. Age-matched L-selectin-deficient $(n=5)$ and wild type mice $(n=3) 42-46$ wk old were given an overdose of inhaled halothane. The chest and abdomen were rapidly opened, the base of the heart was ligated to prevent escape of the pulmonary blood volume, and a blood sample from the inferior vena cava was obtained for measurement of circulating leukocyte and neutrophil counts using a hemocytometer and blood smears. The thoracic organs were removed en bloc, and a $2.5 \%$ glutaraldehyde (in sodium cacodylate) buffer was instilled through the trachea at a pressure of $25 \mathrm{~cm} \mathrm{H}_{2} \mathrm{O}$. Sections (1-2 $\mathrm{mm}^{3}$ cubes) were cut from the peripheral lung tissue of the left middle lobe, post-fixed in $1 \% \mathrm{OsO}_{4}$, and embedded in epoxy resin (Epox 812; Ernest F. Fullan, Inc., Latham, NY). Thin (70-90 nm) sections were cut, stained with uranyl acetate and lead citrate, and examined using a transmission electron microscope (Gloeilampfabrieken; Phillips, Einhoven, The Netherlands). Randomly selected fields of alveolo-capillary walls were sampled by photographing each grid square. In 36 micrographs from each animal, the numbers of intracapillary neutrophils and $\mathrm{RBC}$ were counted, and the results were expressed as the number of intracapillary neutrophils/100 RBC.

Neutrophil sequestration induced by intravascular complement fragments. Zymosan-activated plasma (ZAP) was used as a source of complement fragments and was prepared as previously described (19). In brief, zymosan A yeast (Z-4250; Sigma Chemical Co., St. Louis, MO) was boiled for $15 \mathrm{~min}$ and centrifuged twice at 1,200 $\mathrm{g}$. Heparinized blood obtained from donor L-selectin deficient or wild type mice was centrifuged at $1,200 \mathrm{~g}$. The plasma was incubated with the boiled zymosan yeast $(5 \mathrm{mg} / \mathrm{ml}$ plasma $)$ for $30 \mathrm{~min}$ at $37^{\circ} \mathrm{C}$. The ZAP was centrifuged twice at $1,200 \mathrm{~g}$ for $10 \mathrm{~min}$ to remove the yeast. ZAP was always used within $1 \mathrm{~h}$ of its preparation, and each mouse received plasma from a mouse with a similar genotype.

Age-matched L-selectin $(n=15)$ and wild type mice $(n=15) 10$ 14 wk old were anesthetized with ketamine hydrochloride (80-100 $\mathrm{mg} / \mathrm{kg}$ i.m.) and acepromazine maleate (5-10 $\mathrm{mg} / \mathrm{kg}$ i.m.). ZAP (50 $\mu \mathrm{l} /$ mouse) was injected intravenously. After either 1 or $5 \mathrm{~min}$, the mice received an overdose of inhaled halothane. Control L-selectin deficient and wild type mice received intravenous injection of murine plasma. The chest and abdomen were rapidly opened, the base of the heart was ligated to prevent escape of the pulmonary blood volume, and a blood sample from the inferior vena cava was obtained for measurement of circulating leukocyte and neutrophil counts using a hemocytometer and blood smears. The thoracic organs were removed en bloc, and $6.0 \%$ glutaraldehyde in phosphate buffer was instilled through the trachea at a pressure of $25 \mathrm{~cm} \mathrm{H}_{2} \mathrm{O}$.

Tissue sections were cut through the midportion of both lungs, and paraffin sections $(5-7 \mu \mathrm{m})$ were prepared. The size of the neutrophil accumulation within the alveolar capillaries was quantitated by counting the number of neutrophils in 10 randomly selected fields of alveolar walls. The number of leukocytes within the noncapillary microvasculature, both arterioles and venules, was quantitated by counting the number of cells in each vessel and measuring the luminal surface area. This surface area was determined by outlining the endothelium of all vessels within the section that measured 20-100 $\mu \mathrm{m}$ using a microscope with a drawing tube and a digitizing pad interfaced with PC-driven morphometry software (SigmaScan version 2.0; Jandel Scientific Software, San Rafael, CA). The intravascular neu- 
trophils were categorized as free of interactions with the endothelium, touching (but not spread along) the endothelium, or spread (flattened) along the endothelium. The data are expressed as the number of free, touching, or spread neutrophils $/ 1,000 \mu \mathrm{m}^{2}$ vessel lumen.

Neutrophil emigration during bacterial pneumonia. S. pneumoniae and E. coli were grown overnight on trypticase soy agar plates with $5 \%$ sheep RBCs. The organisms were suspended in saline, and the concentrations were measured using a spectrophotometer and by quantitative cultures to determine the number of colony forming units.

Age-matched L-selectin-deficient $(n=10)$ and wild type mice $(n=10)$ at $12-16 \mathrm{wk}$ of age were anesthetized with ketamine hydrochloride (80-100 mg/kg i.m.) and acepromazine maleate $(5-10 \mathrm{mg} / \mathrm{kg}$ i.m.). The trachea was exposed through a ventral midline incision. ${ }^{125} \mathrm{I}$-labeled albumin $(0.1 \mu \mathrm{Ci} /$ mouse $)$ was injected intravenously to estimate the accumulation of extravascular edema $(27,28)$. Either $S$. pneumoniae $\left(50 \mu \mathrm{l}\right.$ of a suspension of $1-5 \times 10^{9} \mathrm{CFU} / \mathrm{ml}$ saline) or $E$. coli $\left(50 \mu \mathrm{l}\right.$ of a suspension of $1-3 \times 10^{7} \mathrm{CFU} / \mathrm{ml}$ saline) mixed with $5 \%$ colloidal carbon was instilled into the lungs through a 24 gauge angiocatheter $15 \mathrm{~min}$ later. After $5 \mathrm{~h}$ and $58 \mathrm{~min},{ }^{51} \mathrm{Cr}-\mathrm{RBC}(0.25-0.30$ $\mu \mathrm{Ci} /$ mouse) were injected intravenously to measure pulmonary intravascular blood volume. At $6 \mathrm{~h}$, the mice received an inhaled overdose of halothane. The base of the heart was tied, a blood sample was obtained for measurement of radioisotope levels, and the lungs were fixed and sectioned as described above. A plasma sample was prepared, and the lungs, blood sample, and plasma sample were placed in preweighted vials, reweighed, and counted in a gamma counter (Cobra Quantum; Packard Instrument Company, Meriden, CT) interfaced with software to correct for multiple isotopes.

Edema formation, as measured by quantitation of extravascular albumin (EVA), was calculated by $(27,28)$ :

$\mathrm{EVA}=\left(\right.$ total $^{125} \mathrm{I}$-albumin volume in lung $)-$

(intravascular ${ }^{125} \mathrm{I}$-albumin volume)

where:

total ${ }^{125} \mathrm{I}$-albumin volume in lung $=\left({ }^{125} \mathrm{I}\right.$-albumin in lung $) /$

( ${ }^{125} \mathrm{I}$-albumin/g plasma),

and

intravascular ${ }^{125} \mathrm{I}$-albumin volume $=\left({ }^{51} \mathrm{Cr}-\mathrm{RBC}\right.$ in lung $) /$

$\left({ }^{51} \mathrm{Cr}-\mathrm{RBC} / \mathrm{g}\right.$ blood $) \times(1-$ hematocrit $)$

Both the size of the total (intravascular and extravascular) neutrophil accumulation within the alveolar walls and spaces and the

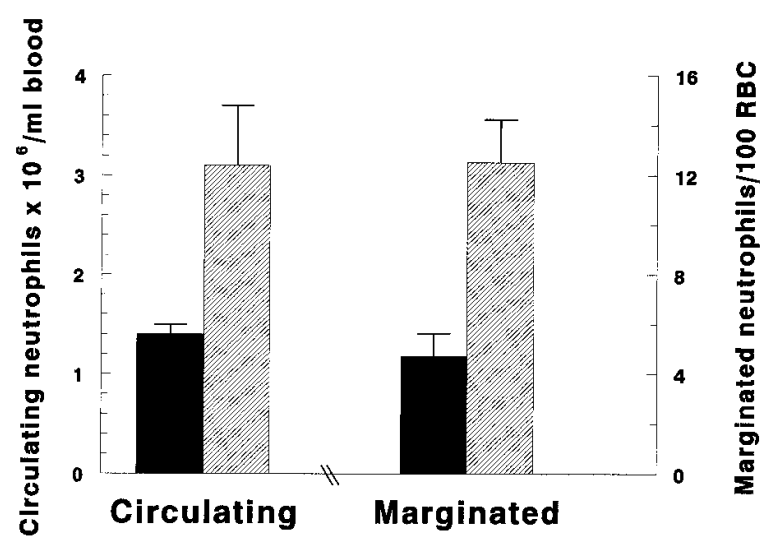

Figure 1. Circulating neutrophils and neutrophils marginated within the lungs of mice without experimentally induced inflammation. L-selectin-deficient mice ( $n=5$, striped bars) showed a 2.2-fold increase in the number of circulating neutrophils compared to wild type mice $(n=3$, dark bars, $P<0.01)$. This increase was paralleled by a similar increase in the size of the marginated neutrophil pool of 2.4fold $(P<0.01)$. number of emigrated (intraalveolar) neutrophils was quantitated. The size of the total accumulation was measured by reflecting a grid onto the microscopic field using a microscope with a drawing tube and counting the number of dots that fell on neutrophils in five randomly selected fields of peripheral lung tissue that contained colloidal carbon within the alveolar spaces (28). These data are expressed as the percent of the alveolar space that was occupied by neutrophils. The number of emigrated neutrophils was quantitated by counting the number of neutrophils present within all alveolar spaces in randomly selected fields containing colloidal carbon until 200 alveolar spaces were counted (22). The data are expressed as the number of emigrated neutrophils/100 alveolar spaces. The number of spread, touching, and free neutrophils in the noncapillary small vessels were quantitated as described above.

Statistics. Analyses of variance or Student's $t$ test were used to compare circulating leukocyte and neutrophil counts, the accumulation of neutrophils in the capillaries and noncapillary microvessels, the emigration of neutrophils, and EVA as appropriate. The modified Bonferonni correction was used to correct for multiple comparisons when significant overall differences were identified in the analysis of variance (29). A probability of less than 0.05 for the null hypothesis was accepted as indicating a statistically significant difference. Data are expressed as mean \pm SEM.

\section{Results}

Neutrophil margination in the pulmonary capillaries. In normal mice without experimentally induced inflammation, the circulating leukocyte count measured $5.1 \pm 0.8 \times 10^{6} / \mathrm{ml}$ in the L-selectin-deficient mice, significantly higher than the value of $3.1 \pm 1.4 \times 10^{6} / \mathrm{ml}$ observed in wild type mice $(P<0.05)$. This increase was due entirely to an increase in the number of circulating neutrophils (Fig. 1). The number of neutrophils that were marginated within the pulmonary capillaries was also significantly increased in the L-selectin-deficient mice (Fig. 1). The increase in the size of the marginated pool (2.4-fold) exactly paralleled the increase in the circulating neutrophil counts (2.2-fold).

Neutrophil sequestration induced by intravascular complement fragments. Both the number of circulating neutrophils in blood samples from the inferior vena cava (Fig. 2) and the

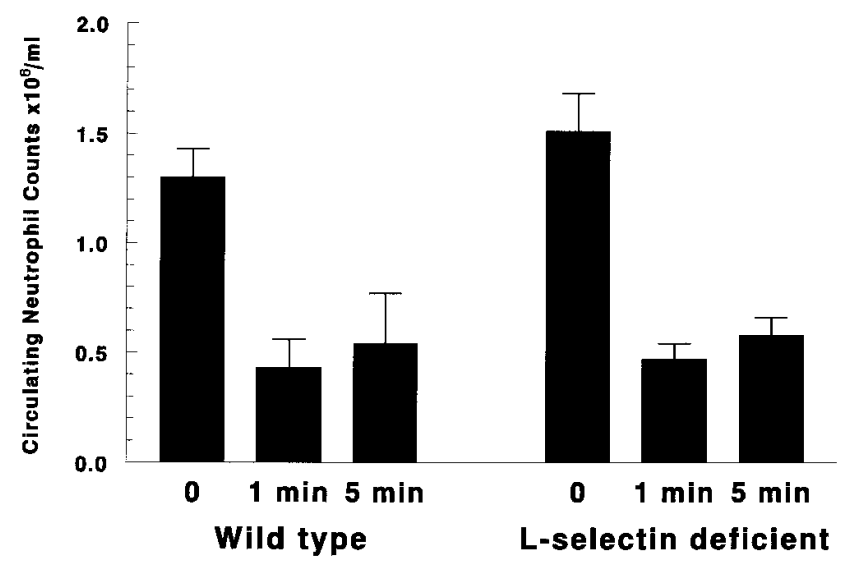

Figure 2. The number of circulating neutrophils in the blood of mice given intravenous injection of either plasma (0) or complement fragments for 1 or $5 \mathrm{~min}$. Complement fragments induced a significant decrease in the circulating neutrophil counts at both 1 and 5 min after injection that was similar in both the L-selectin-deficient and wild type mice $(P<0.01$ compared to mice with the same phenotype at time 0$) . n=5$ in each group. 


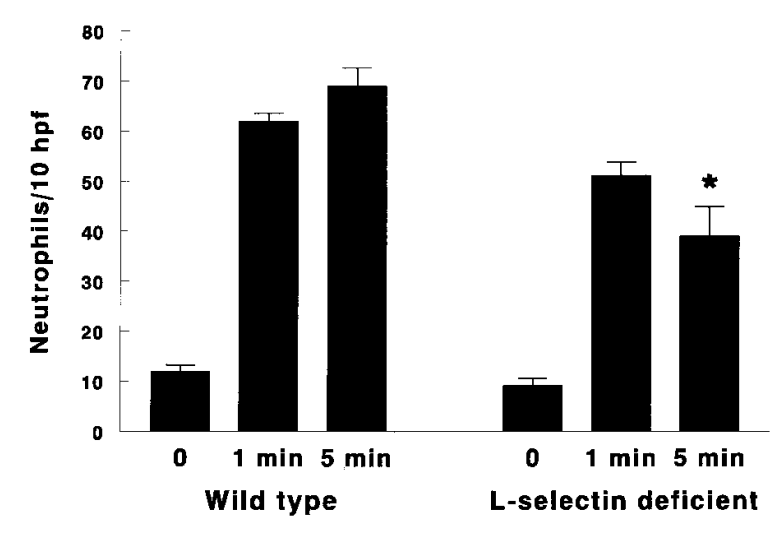

Figure 3. The number of neutrophils in the pulmonary capillaries of mice given intravenous injection of either plasma (0) or complement fragments for 1 or $5 \mathrm{~min}$. Complement fragments induced a significant increase in the number of intracapillary neutrophils sequestered in the lungs within $1 \mathrm{~min}$ of injection $(P<0.01$ compared to time 0$)$. This increase was similar in both the L-selectin-deficient and wild type mice. However, the number of intracapillary neutrophils was significantly less after $5 \mathrm{~min}$ in the L-selectin-deficient compared to wild type mice ( $* P<0.05$ compared to wild type mice at the same time). $n=5$ in each group.

number of neutrophils within the pulmonary capillaries (Fig. 3) was similar in L-selectin-deficient and wild type mice that did not receive complement fragments. Injection of complement fragments induced a fall in the circulating neutrophil counts at both 1 and 5 min after injection that was similar in the L-selectin-deficient and the wild type mice (Fig. 2). This decrease was associated with an increase in the number of intracapillary neutrophils within the lung (Fig. 3) that was simi-

Table I. Spread, Touching, and Free Leukocytes in Noncapillary Microvessels after Injection of Complement Fragments

\begin{tabular}{lll}
\hline & Wild type Mice & L-selectin-deficient mice \\
\hline No complement fragments & & \\
$\quad$ Total & $4.7 \pm 0.5$ & $5.0 \pm 0.4$ \\
Free & $0.1 \pm 0.1$ & $0.5 \pm 0.1$ \\
$\quad$ Touching & $2.0 \pm 0.4$ & $1.4 \pm 0.2$ \\
$\quad$ Spread & $2.6 \pm 0.3$ & $3.1 \pm 0.2$ \\
min after injection & & \\
$\quad$ Total & $5.6 \pm 0.6$ & $3.9 \pm 0.4$ \\
Free & $0.4 \pm 0.1$ & $0.2 \pm 0.1$ \\
Touching & $1.5 \pm 0.3$ & $1.0 \pm 0.2$ \\
$\quad$ Spread & $3.7 \pm 0.4^{*}$ & $2.7 \pm 0.2$ \\
5 min after injection & & \\
Total & $5.9 \pm 0.5$ & $5.4 \pm 0.5$ \\
Free & $0.6 \pm 0.2$ & $0.7 \pm 0.1$ \\
Touching & $1.3 \pm 0.2$ & $1.6 \pm 0.3$ \\
Spread & $4.0 \pm 0.4^{*}$ & $3.2 \pm 0.3$ \\
& & \\
\hline
\end{tabular}

Mean \pm SEM number of leukocytes $/ 1,000 \mu \mathrm{m}^{2}$ vessel lumen. $n=5$ in each group. *Significantly different from the same value in the pulmonary capillary microvessels of mice that did not receive injection of complement fragments and from the same value in the L-selectin-deficient mice $(P<0.05)$.
Table II. Circulating Neutrophil Counts 6 h after Instillation of Bacteria

\begin{tabular}{lcc}
\hline & Wild type mice & $\begin{array}{c}\text { L-selectin-deficient } \\
\text { mice }\end{array}$ \\
\hline No pneumonia & $0.8 \pm 0.3$ & $0.9 \pm 0.4$ \\
E. coli-induced pneumonia & $1.0 \pm 0.3$ & $1.1 \pm 0.3$ \\
S. pneumoniae-induced pneumonia & $1.1 \pm 0.4$ & $1.1 \pm 0.3$ \\
\hline
\end{tabular}

Data expressed as the mean \pm SEM $\times 10^{6} / \mathrm{ml} . n=5$ in each group.

lar in magnitude at $1 \mathrm{~min}$ in L-selectin-deficient and wild type mice (Fig. 3). However, the number of intracapillary neutrophils was significantly less in the L-selectin deficient mice than wild type mice at 5 min after injection (Fig. 3), suggesting that L-selectin was required to retain the sequestered neutrophils within the lung.

In the arterioles and venules of mice that did not receive injections of complement fragments, no significant differences in the total number of leukocytes or in the number of free, touching or spread leukocytes were observed between L-selectindeficient and wild type mice (Table I). Injection of complement fragments significantly increased the numbers of spread neutrophils in the noncapillary microvessels of wild type mice at both 1 and $5 \mathrm{~min}$ (Table I). However, this increase was not observed in the L-selectin-deficient mice. Intravascular complement fragments did not significantly increase the number of touching or free neutrophils in either wild type or L-selectindeficient mice at 1 or $5 \mathrm{~min}$ after injection (Table I).

Neutrophil emigration during bacterial pneumonia. The circulating neutrophil counts were similar in L-selectin-deficient and wild type mice $6 \mathrm{~h}$ after instillation of organisms (Table II).

The total accumulation of neutrophils within the intra- and extravascular space in response to E. coli within the airways was smaller in the pneumonic regions of L-selectin-deficient mice than wild type mice (Fig. 4). In contrast, no significant defect in the number of accumulated neutrophils was observed after instillation of S. pneumoniae (Fig. 4).

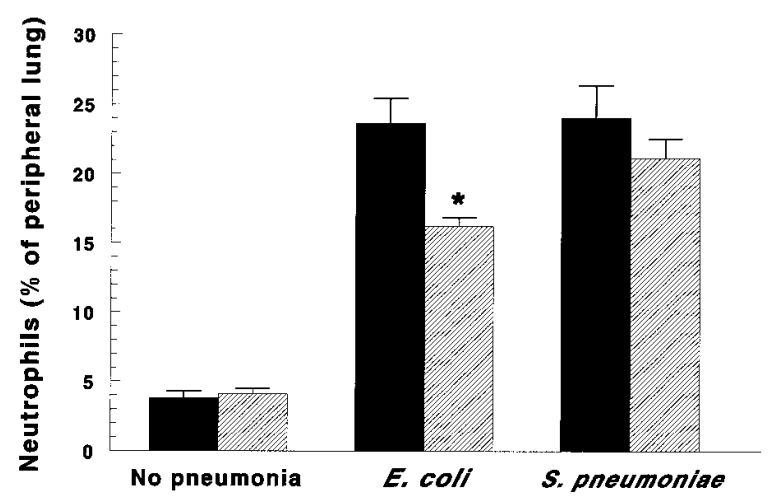

Figure 4. The total accumulation of neutrophils within the regions of pneumonia. Either E. coli or S. pneumoniae induced an increase in the total number of neutrophils within the peripheral lung (alveolocapillary walls and the alveolar space) in both wild type (dark bars) and L-selectin-deficient (striped bars) mice. However, this increase was significantly less in the E. coli-induced pneumonias in the L-selectin-deficient mice than in the wild type mice $(* P<0.05)$. $n=5$ in each group. 


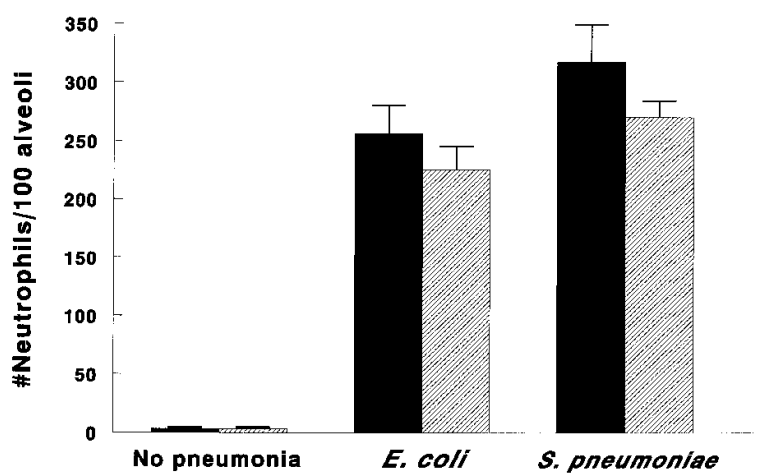

Figure 5. The number of emigrated neutrophils within the regions of pneumonia. Either E. coli or S. pneumoniae induced an increase in the number of neutrophils that emigrated into the alveolar spaces that was similar in wild type (dark bars) and L-selectin-deficient (striped bars) mice. $n=5$ in each group.

Despite the defect in the total number of accumulated neutrophils, the number of neutrophils that emigrated into the alveolar space in response to either $E$. coli or $S$. pneumoniae was similar in L-selectin-deficient and wild type mice (Fig. 5). The amount of edema formation, as quantitated by measurement of EVA, induced by either organism was also similar (Fig. 6).

The numbers of spread, touching, free, and total neutrophils within the lumen of the pulmonary noncapillary microvasculature in uninfected mice and mice with either $E$. coli or $S$. pneumoniae-induced pneumonia are shown in Table III. There were no significant differences in these values between the control L-selectin-deficient and wild type mice that did not receive intratracheal instillation of organisms. In E. coli-induced pneumonia, the number of neutrophils touching the endothelium was increased in wild type mice compared to that in uninfected mice. However, this increase was not observed in L-selectin-deficient mice, indicating that L-selectin-deficient mice demonstrated a defect in neutrophil adhesion within the noncapillary microvessels. In S. pneumoniae-induced pneumonia, the number of spread (but not touching) neutrophils was significantly increased in wild type mice after infection. This increase did not occur in L-selectin-deficient mice (Table III).

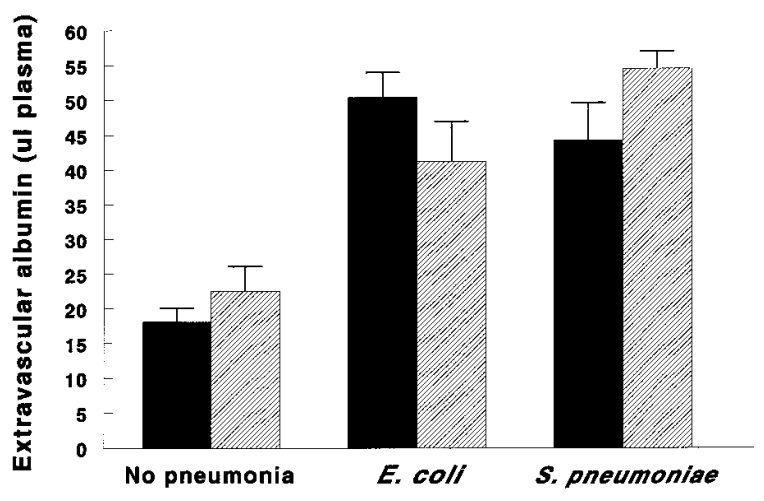

Figure 6. Edema formation during bacterial pneumonia. Either $E$. coli or $S$. pneumoniae induced an increase in the accumulation of EVA that was similar in wild type (dark bars) and L-selectin-deficient (striped bars) mice. $n=5$ in each group.
Table III. Spread, Touching, and Free Leukocytes in Noncapillary Microvessels 6 h after Instillation of Bacteria

\begin{tabular}{lcc}
\hline & Wild type mice & L-selectin-deficient mice \\
\hline No pneumonia & & \\
$\quad$ Total & $3.5 \pm 0.6$ & $3.7 \pm 0.5$ \\
Free & $0.3 \pm 0.4$ & $0.4 \pm 0.2$ \\
Touching & $1.0 \pm 0.3$ & $1.0 \pm 0.2$ \\
$\quad$ Spread & $2.2 \pm 0.4$ & $2.3 \pm 0.5$ \\
E. coli-induced pneumonia & \\
Total & $5.3 \pm 1.1^{*}$ & $4.1 \pm 0.5$ \\
Free & $0.4 \pm 0.1$ & $0.3 \pm 0.1$ \\
Touching & $2.0 \pm 0.4^{*}$ & $1.4 \pm 0.3$ \\
Spread & $2.9 \pm 0.7$ & $2.5 \pm 0.3$ \\
S. pneumoniae-induced pneumonia & \\
Total & $4.6 \pm 0.6$ & $3.6 \pm 0.2$ \\
Free & $0.2 \pm 0.2$ & $0.3 \pm 0.2$ \\
Touching & $1.3 \pm 0.4$ & $1.0 \pm 0.3$ \\
Spread & $3.0 \pm 0.3^{*}$ & $2.4 \pm 0.2$ \\
& &
\end{tabular}

Mean \pm SEM number of leukocytes $/ 1,000 \mu \mathrm{m}^{2}$ vessel lumen. $n=5$ in each group. $*$ Significantly different from the same value in the pulmonary capillary microvessels of mice that did not receive instillation of organisms $(P<0.05)$.

\section{Discussion}

This study investigated the role of L-selectin in neutrophil margination within normal lungs, neutrophil sequestration induced by intravascular complement fragments, and neutrophil emigration induced by bacterial pneumonias. The results show interesting and specific functions for L-selectin in sequestration and emigration but no role for L-selectin in neutrophil margination. There are, however, striking differences in the function of L-selectin within the pulmonary compared to the systemic microvasculature.

L-selectin does not play a role in the formation of the marginated pool of neutrophils within normal, uninflamed pulmonary microvasculature, either the capillaries or the arterioles and venules. In fact, the number of marginated neutrophils increased more than twofold in the L-selectin-deficient mice compared with wild type mice. However, this increase exactly paralleled the increase observed in the number of circulating neutrophils, suggesting that, at least for small changes in the circulating pool size, the marginated and circulating pool sizes were directly proportional. In addition, the L-selectin-deficient mice that received plasma instead of complement fragments also showed no defects in the size of the marginated pool, and the sizes of both the circulating and marginated pools were similar to those of age-matched wild type mice (Figs. 2 and 3). L-selectin also played no role in the number of neutrophils within the noncapillary microvasculature, as the number of spread, touching, and free neutrophils was similar in L-selectin-deficient and wild type mice that did not receive complement fragments (Table I).

Circulating neutrophil counts may vary in L-selectin-deficient mice as a function of aging. Neutrophil counts were increased in the group of L-selectin-deficient mice in which the marginated pool was quantitated ultrastructurally compared to age-matched wild type mice (Fig. 1). These mice were 42-46 
wk old at the time of this experiment. In contrast, the counts in the L-selectin deficient mice given complement fragments were not increased (Fig. 2). These mice were younger (10-14 wk). No significant differences in the circulating neutrophil counts were observed between young and old wild type mice. These data suggest that this difference may be due to a gradual increase in the neutrophil counts with age in the L-selectindeficient mice that does not occur in wild type mice. This increase in circulating neutrophil counts has not been consistently observed in mice housed in a specific pathogen-free barrier facility (25). Nonetheless, these data further demonstrate that when circulating neutrophil counts increase in L-selectin-deficient mice, the size of the marginated pool increases proportionally. These observations support our conclusion the L-selectin is not a major contributor to the margination of neutrophils.

L-selectin was not required for the initial sequestration of neutrophils within the pulmonary capillaries observed within 1 min after injection of complement fragments, as the decrease in the circulating neutrophil counts and the increase in the intracapillary neutrophils was similar in the L-selectin deficient and wild type mice. However, at $5 \mathrm{~min}$, there were significantly fewer intracapillary neutrophils in the lungs of L-selectin-deficient mice, suggesting that interactions between L-selectin and its endothelial ligand contributed to maintaining the sequestered neutrophils within the pulmonary capillaries. This role for L-selectin-mediated interactions is similar to the role observed for CD11/CD18, which is also not needed to sequester neutrophils within the lungs but is required to keep sequestered neutrophils within the lungs for more than 4 min (19). Whether interactions between L-selectin and its ligand are a prerequisite for CD11/CD18-mediated adhesion or whether L-selectin and CD11/CD18 are utilized in tandem cannot be determined from these studies. However, because rolling of neutrophils along the capillary endothelium does not occur, most likely due to spatial constraints $(10,15)$, the function of L-selectin is likely to be different from that observed in the postcapillary venules of the systemic circulation.

Injection of complement fragments induced an increase in the number of leukocytes that were flattened and spread along the endothelium of arterioles and venules. In contrast to the role of L-selectin in neutrophil sequestration within the pulmonary capillaries, L-selectin-deficient mice showed a complete defect in the increase in neutrophil spreading. These data suggest that L-selectin was critically required at both 1 and 5 min for the firm attachment of neutrophils along the endothelium of these noncapillary microvessels. It is possible that this difference in spreading in L-selectin deficient mice relates to a signal transduction role for L-selectin in leukocyte-endothelial interactions (D. Steeber and T. Tedder, unpublished data; 30). Without the L-selectin-mediated interaction, the appropriate signals for firm adhesion and spreading appear not to be generated. Previous studies have shown that the number of neutrophils that accumulate in noncapillary microvessels is small, measuring only $4 \%$ of the total number of sequestered neutrophils compared to $96 \%$ which sequester within the capillaries $(19,31)$. However, the contribution of neutrophils sequestered within capillaries compared to that within the noncapillary microvessels to the increased vascular permeability that develops in this injury (32) and other more severe injuries has not been established.

In pneumonia, both sequestration of neutrophils within the pulmonary microvasculature and emigration of neutrophils into the lung parenchyma are induced (33). In this study, the total accumulation and the number of emigrated neutrophils was evaluated. L-selectin-deficient mice showed a small but significant defect of $40 \%$ in the total accumulation of neutrophils within $E$. coli-induced pneumonia. In contrast, no significant difference was observed when $S$. pneumoniae was the stimulus. However, no defect in neutrophil emigration into the alveolar spaces was observed in response to either stimulus. These data suggest that while L-selectin may play a role in the intravascular accumulation of neutrophils when induced by $E$. coli, a stimulus eliciting CD11/CD18-dependent emigration, there is no resultant defect in emigration through either CD11/ CD18-dependent or -independent pathways. Previous studies demonstrated that only a small fraction (2-3\%) of the marginated neutrophils actually emigrate into the alveolar space (33). Taken together, these studies suggest that L-selectin partially mediates the intravascular accumulation of neutrophils in response to E. coli, but inhibition of this function is not sufficient to cause a defect in the number of neutrophils that emigrate into the alveolar space and destroy the organisms. As mentioned above, the role of L-selectin in the accumulation of neutrophils within capillaries, where rolling does not occur (10), is unclear, and mediation of loose interactions without true rolling may be its function. Finally, these studies also indicate that L-selectin plays no role in either intravascular sequestration, emigration, or edema formation in response to $S$. pneumoniae, a stimulus inducing CD11/CD18-independent adhesion pathways, suggesting that L-selectin does not mediate CD11/CD18independent pathways of emigration.

Although the major site of neutrophil sequestration and emigration in bacterial pneumonia, and in inflammation induced by other inflammatory stimuli within the distal lung parenchyma, is thought to be the pulmonary capillaries (34), neutrophils also accumulate within the pulmonary arterioles and venules. In $E$. coli-induced pneumonia, an increase in the total number of leukocytes within the noncapillary microvessels was observed, and this increase was due primarily to increased numbers of leukocytes touching endothelium and a tendency toward increases in spread neutrophils. In S. pneumoniaeinduced pneumonia, the increase was observed only in the number of spread leukocytes. In L-selectin-deficient mice, the increases induced by $E$. coli were partially prevented and the increases induced by $S$. pneumoniae were completely absent. These data indicate that L-selectin was required for either increase.

Spreading appears to correspond to the phenomenon of firm adhesion, as observed by videomicroscopic studies of systemic postcapillary venules, while touching may correspond to either tethering to the endothelium through rolling or increased proximity to the endothelial surface due to either increased numbers of neutrophils or altered hemodynamic factors. It is unclear why $E$. coli induced primarily an increase in touching while either $S$. pneumoniae or intravascular complement fragments induced only an increase in spreading. However, these data suggest that the time spent tethered to the endothelium may vary depending on the stimulus. Tethering and spreading may occur very rapidly when induced by $S$. pneumoniae or complement fragments, resulting in no increase in tethering when evaluated by static histological techniques. While intravascular complement fragments induce prolonged neutrophil sequestration that requires CD11/CD18 (19), neutrophil 
emigration induced by intraairway instillation of this stimulus does not (35). It is interesting to postulate that the time leukocytes spend tethered to endothelium is shorter when CD11/ CD18-independent pathways of emigration are utilized than when emigration requires CD11/CD18-mediated adhesion.

These studies raise questions about possible ligands for the L-selectin expressed on neutrophils. No ligand for neutrophil L-selectin on pulmonary endothelial cells of either arterioles, capillaries, or venules has yet been identified. Because L-selectin is utilized within 5 min after injection of complement fragments, the ligand is likely to be either constitutively expressed or rapidly upregulated from granular pools. Mulligan et al. have demonstrated a role for neutrophil L-selectin in lung injuries induced by either cobra venom factor or IgG immune complexes $(11,12)$. Cobra venom factor-induced lung injury required only $30 \mathrm{~min}$, also suggesting that the ligand does not require protein synthesis for expression. Alternatively, the L-selectin ligand may be located on neutrophils and mediate homotypic aggregation $(30,36)$. However, previous studies have shown that both the large number of neutrophils sequestered within the capillaries and the small number accumulating in small noncapillary vessels were primarily single (31). These data suggest that homotypic aggregation is not a prominent mechanism through which complement fragment-induced neutrophil sequestration occurs.

In summary, L-selectin does not play a role in margination of neutrophils within capillaries, arterioles, or venules of uninflamed lungs. It is not required in the initial events that result in sequestration of neutrophils induced by intravascular complement fragments, which is most likely mediated through stimulus-induced changes in the biomechanical properties of neutrophils, decreasing their ability to deform and preventing their passage through the pulmonary capillary bed $(13,14$, 19-21, 37). However, L-selectin plays an important role similar to that of CD11/CD18 in maintaining the sequestered neutrophils within the capillaries and in the accumulation of neutrophils within the noncapillary microvessels. During bacterial pneumonias, L-selectin plays a role in the accumulation of neutrophils within noncapillary microvessels in response to either E. coli or S. pneumoniae and within capillaries in response to $E$. coli but not $S$. pneumoniae, but is not required for emigration of neutrophils out of the microvasculature or the development of edema in response to either organism, as evaluated using L-selectin-deficient mice. These studies suggest that L-selectin plays a role in pulmonary inflammation, particularly when CD11/CD18-mediated pathways of sequestration or emigration are utilized.

\section{Acknowledgements}

The authors would like to thank Dr. James C. Hogg for innumerable valuable discussions.

These studies were supported by Public Health Service HL48160, HL-52466, and HL-33009 to C.M. Doerschuk and CA-54464, HL-50985, and AI-26872 to T.F. Tedder. Dr. Tedder is a Stohlman Scholar of the Leukemia Society of America. C.M. Doerschuk is the recipient of a Career Investigator Award from the American Lung Association.

\section{References}

1. Carlos, T.M., and J.M. Harlan. 1994. Leukocyte-endothelial adhesion molecules. Blood. 84:2068-2101.
2. Tedder, T.F., D.A. Steeber, A. Chen, and P. Engel. 1995. The selectins: vascular adhesion molecules. FASEB J. 9:866-873.

3. Ley, K., and T.F. Tedder. 1995. Leukocyte interactions with vascular endothelium: new insights into selectin-mediated attachment and rolling. $\mathrm{J}$. Immunol. 155:525-528.

4. Ma, X.-L., A.S. Weyrich, D.J. Lefer, M. Buerke, K.H. Albertine, T.K Kishimoto, and A.M. Lefer. 1993. Monoclonal antibody to L-selectin attenuates neutrophil accumulation and protects ischemic reperfused cat myocardium. Circulation. 88:649-658.

5. Jutila, M.A., E.L. Berg, T.K. Kishimoto, L.J. Picker, R.F. Bargatze, D.K. Bishop, C.G. Orosz, N.W. Wu, and E.C. Butcher. 1989. Inflammation-induced endothelial cell adhesion to lymphocytes, neutrophils, and monocytes: role of homing receptors and other adhesion molecules. Transplantation (Baltimore) 48:727-731.

6. Watson, S.R., C. Fennie, and L.A. Lasky. 1991. Neutrophil influx into an inflammatory site inhibited by a soluble homing receptor-IgG chimaera. Nature (Lond.). 349:164-167.

7. Lewisohn, D.M., R.F. Bargatze, and E.C. Butcher. 1987. Leukocyte-endothelial cell recognition: evidence of a common molecular mechanism shared by neutrophils, lymphocytes, and other leukocytes. J. Immunol. 138:4313-4321.

8. Yang, X.D., N. Karin, R. Tisch, L. Steinman, and H.O. McDevitt. 1993. Inhibition of insulinitis and prevention of diabetes in nonobese diabetic mice by blocking L-selectin and very late antigen 4 adhesion receptors. Proc. Natl. Acad. Sci. USA. 90:10494-10498.

9. Turunen, J.P., T. Paavonen, M.L. Majuri, S. Tiisala, P. Mattila, A. Mennander, C.G. Gahmberg, P. Hayry, T. Tamatani, M. Miyasaka, et al., 1994. Sialyl Lewis ${ }^{\mathrm{x}}$ - and L-selectin-dependent site-specific lymphocyte extravasation into renal transplants during acute rejection. Eur. J. Immunol. 24:1130-1136.

10. Gebb, S.A., J.A. Graham, C.C. Hanger, P.S. Godbey, R.L. Capen, C.M. Doerschuk, and W.W. Wagner, Jr. 1995. Sites of leukocyte sequestration in the pulmonary microcirculation. J. Appl. Physiol. 79:493-497.

11. Mulligan, M.S., S.R. Watson, C. Fennie, and P.A. Ward. 1994. Protective effects of chimeras in neutrophil-mediated lung injury. J. Immunol. 151: 6410-6417.

12. Mulligan, M.S., M. Miyasaka, T. Tamatani, M.J. Jones, and P.A. Ward. 1994. Requirements for L-selectin in neutrophil-mediated lung injury in rats. $J$. Immunol. 153:832-840.

13. Hogg, J.C. 1987. Neutrophil kinetics and lung injury. Physiol. Rev. 67: 1249-1295.

14. Hogg, J.C., and C.M. Doerschuk. 1995. Leukocyte traffic through the lung. Annu. Rev. Physiol. 57:97-114.

15. Doerschuk, C.M., N. Beyers, H.O. Coxson, B. Wiggs, and J.C. Hogg. 1993. Comparison of neutrophil and capillary diameters and their relation to neutrophil sequestration in the lung. J. Appl. Physiol. 74:3040-3045.

16. Lien, D.C., W.W. Wagner, Jr., R.L. Capen, C. Haslett, W.L. Hanson, S.E. Hofmeister, P.M. Henson, and G.S. Worthen. 1987. Physiologic neutrophil sequestration in the canine pulmonary circulation. J. Appl. Physiol. 62:12361243 .

17. Yoder, M.C., L.L. Checkley, U. Giger, W.L. Hanson, K.R. Kirk, R.L. Capen, and W.W. Wagner Jr. 1990. Pulmonary microcirculatory kinetics of neutrophils deficient in leukocyte adhesion-promoting glycoproteins. J. Appl. Physiol. 69:207-213.

18. Mulligan, M.S., M.J. Polley, R.J. Bayer, M.F. Nunn, J.C. Paulson, and P.A. Ward. 1992. Neutrophil-dependent acute lung injury: Requirement for P-selectin (GMP-140). J. Clin. Invest. 90:1600-1607.

19. Doerschuk, C.M. 1992. The role of CD18-mediated adhesion in neutrophil sequestration induced by infusion of activated plasma in rabbits. Am. J. Respir. Cell Mol. Biol. 7:140-148.

20. Worthen, G.S., B. Schwab, E.L. Elson, and G.P. Downey. 1989. Mechanics of stimulated neutrophils: cell stiffening induces retention in capillaries. Science (Wash. DC). 245:183-185.

21. Inano, H., D. English, and C.M. Doerschuk. 1992. Effect of zymosanactivated plasma on the deformability of rabbit polymorphonuclear leukocytes and the role of the cytoskeleton. J. Appl. Physiol. 73:1370-1376.

22. Doerschuk, C.M., R.K. Winn, H.O. Coxson, and J.M. Harlan. 1990. CD18-dependent and -independent mechanisms of neutrophil adherence in the pulmonary and systemic microvasculature of rabbits. J. Immunol. 114:23272333.

23. Arbones, M.L., D.C. Ord, K. Ley, H. Ratech, C. Maynard-Curry, G. Otten, D.J. Capon, and T.F. Tedder. 1994. Lymphocyte homing and leukocyte rolling and migration are impaired in L-selectin-deficient mice. Immunity. 1:247-260.

24. Tedder, T.F., D.A. Steeber, and P. Pizcueta. 1995. L-selectin-deficient mice have impaired leukocyte recruitment into inflammatory sites. J. Exp. Med. 184:2259-2264.

25. Steeber, D.A., N.E. Green, S. Sato, and T.F. Tedder. 1996. Lymphocyte migration in L-selectin-deficient mice: altered subset migration and aging of the immune system. J. Immunol. 157:1096-1106.

26. Ley, K., D.C. Bullard, M.L. Arbones, R. Bosse, D. Vestweber, T.F. Tedder, and A.L. Beaudet. 1995. Sequential contribution of L- and P-selectin to leukocyte rolling in vivo. J. Exp. Med. 181:669-675.

27. Bullard, D.C., L. Qin, I. Lorenzo, W.M. Quinlan, N.A. Doyle, D. Vestweber, C.M. Doerschuk, and A.L. Beaudet. 1995. P-selectin/ICAM-1 dou- 
ble mutant mice: acute emigration of neutrophils into the peritoneum is completely absent but is normal into pulmonary alveoli. J. Clin. Invest. 95:17821788 .

28. Kumasaka, T., W.M. Quinlan, N.A. Doyle, T.P.E. Condon, J. Sligh, F. Takei, A.L. Beaudet, C.F. Bennett, and C.M. Doerschuk. 1996. The role of ICAM-1 in endotoxin-induced pneumonia evaluated using ICAM-1 antisense, anti-ICAM-1 antibodies, and ICAM-1 mutant mice. J. Clin. Invest. 97:23622369.

29. Hollard, B.S., and M.D. Copenhaver. 1987. An improved sequentially rejective Bonferroni test procedure. Biometrics. 43:417-423.

30. Simon, S.I., A.R. Burns, A.D. Taylor, P.K. Gopalan, E.B. Lynam, L.A. Sklar, and C.W. Smith. 1995. L-selectin (CD62L) cross-linking signals neutrophil adhesive functions via the Mac-1 (CD11b/CD18) beta 2-integrin. J. Immunol. 155:1502-1514.

31. Doerschuk, C.M., M.F. Allard, and J.C. Hogg. 1989. Neutrophil kinetics in rabbits during infusion of zymosan-activated plasma. J. Appl. Physiol. 67:8895.

32. Gie, R.P., C.M. Doerschuk, D. English, H.O. Coxson, and J.C. Hogg. 1991. Neutrophil-associated lung injury following the infusion of activated plasma. J. Appl. Physiol. 70:1271-1278.

33. Doerschuk, C.M., J. Markos, H.O. Coxson, D. English, and J.C. Hogg. 1994. Quantitation of neutrophil migration in acute bacterial pneumonia in rabbits. J. Appl. Physiol. 77:2593-2599.

34. Downey, G.P., G.S. Worthen, P.M. Henson, and D.M. Hyde. 1993. Neutrophil sequestration and migration in localized pulmonary inflammation: Capillary localization and migration across the interalveolar septum. Am. Rev. Respir. Dis. 147:168-176.

35. Hellewell, P.G., S.K. Young, P.M. Henson, and G.S. Worthen. 1994 Disparate role of the $\beta_{2}$-integrin CD18 in the local accumulation of neutrophils in pulmonary and cutaneous inflammation in the rabbit. Am. J. Respir. Cell Mol. Biol. 10:391-398.

36. Simon, S.I., Y.P. Rochon, E.B. Lynam, C.W. Smith, D.C. Anderson, and L.A. Sklar. 1993. Beta 2-integrin and L-selectin are obligatory receptors in neutrophil aggregation. Blood. 82:1097-1106.

37. Motosugi, H., L. Graham, T.W. Noblitt, N.A. Doyle, W.M. Quinlan, Y. Li, and C.M. Doerschuk. 1996. Changes in neutrophil actin and shape during sequestration induced by complement fragments in rabbits. Am. J. Pathol. 149: 963-973. 